\title{
Writing Parameters for 3D Refractive Index Structures in Polymethyl Methacrylate Using Femtosecond Radiation at 400 nm
}

\author{
Shijie Liang ${ }^{*}$, Patricia J. Scully ${ }^{*}$ Joerg Schille ${ }^{*, *}$, John Vaughan*, Mohammed Benyezzar", \\ Dun Liu ${ }^{* * *}$, and Walter Perrie ${ }^{* * *}$ \\ * The Photon Science Institute, The University of Manchester, Manchester, UK, M13 9PL \\ E-mail: patricia.scully@manchester.ac.uk \\ ** University of Applied Sciences Mittweida, Technikumplatz 17 in 09648 Mittweida, Germany \\ *** Laser Group, Department of Engineering, The University of Liverpool, Liverpool, UK, L69 3GQ
}

\begin{abstract}
Sub-surface modification by a femtosecond laser, producing pulses of $100 \mathrm{fs}$ duration at $400 \mathrm{~nm}$ wavelength for fabrication of optical components is investigated, within sub-millimetre thick sheets of undoped polymethyl methacrylate (PMMA). For effective structuring, the influence of laser writing parameters and focusing conditions were studied to optimise the pulse energy, pulses spacing and focusing depth in the material. Permanent sub-surface refractive index structures with a minimum feature size $(\sim 3 \mu \mathrm{m})$ at a depth of $\sim 500 \mu \mathrm{m}$ demonstrated the feasibility of writing flexible $3-\mathrm{D}$ microstructures within a thin PMMA substrate. This investigation of focusing depth location in PMMA bulk material enabled photonic structures at the desired spatial resolution to be written within the core of single mode polymer optical fibre under controlled laser writing conditions. A long period fibre grating (LPG) consisting of a $2.8 \mu \mathrm{m}$ wide refractive index structure with a periodicity of $250 \mu \mathrm{m}$ and containing a series of attenuation bands in visible range, demonstrated a photonic structure written into a single mode polymer optical fibre.
\end{abstract}

DOI: $10.2961 / \mathrm{jlmn} .2010 .01 .0015$

Keywords: femtosecond laser, polymethyl methacrylate, PMMA, refractive index modification, microstructure, photonic structure, focusing depth, polymer optical fibre, POF, long period grating, LPG

\section{Introduction}

Femtosecond laser technology forms a powerful tool for rapid prototyping with the capability to form threedimensional structures with reduced feature size in transparent materials via nonlinear photon absorption. Optical structures in transparent materials such as waveguides [1-3], gratings $[4,5]$, and splitters [6] are important components for integrated optical circuits [7, 8], micro-fluidic and labon-a-chip devices $[9,10]$.

Polymethyl methacrylate (PMMA) is of great interest as a photonic material, because it is inexpensive, flexible and biocompatible, and thus ideal for disposable devices for clinical, biological and chemical applications [11]. The low processing temperature of PMMA [12] permits addition of organic dopants (unsuited to dielectrics having high critical temperature $\mathrm{Tc}$, such as glass) to impart optical activity and lasing properties, and allows PMMA to be injection moulded to create structured surfaces and channels for micro-fluidic devices [13, 14].

In 2003, Scully et al [15] demonstrated the first permanent refractive index structures within undoped bulk PMMA, in the form of diffraction gratings, directly written by femtosecond (fs) laser at $800 \mathrm{~nm}, 40 \mathrm{fs}, 1 \mathrm{kHz}$. Permanent refractive index changes, $\Delta \mathrm{n}$, of up to $5 \times 10^{-4}$ were obtained with longevity of several years. Waveguides and gratings within optical fibre were also demonstrated [16]. The photochemical mechanism, as well as the effect of wavelengths and pulse durations on the multi-photon process related to bandgap was elucidated $[16,17]$. Structures with minimum diffraction limited feature size of $0.42 \mu \mathrm{m}$ were achieved within bulk clinical grade PMMA by holographic writing at $387 \mathrm{~nm}$ [18]. However, the relationship of writing depth below the surface of laser modified polymeric material has not been fully investigated to date.

Fabricating small feature size of micro-structure cross section at various depths below the surface of transparent media is challenging, because laser pulse filamentation occurs due to a combination of self-focusing and selfdefocusing effects [19-21], elongating the focal volume along the beam propagation direction. At sufficiently high laser fluences, an irregular structure is formed around the centre of the beam focal point, surrounded by a refractive index modification [22]. Liu et al [23] showed the influence of focusing depth was significant when writing waveguides in fused silica with a $0.5 \mathrm{NA}$ objective. Although the width of the waveguide cross section remained $\sim 3 \mu \mathrm{m}$, the cross-sectional depth increased from 4 to $12 \mu \mathrm{m}$ in writing from 300 to $2100 \mu \mathrm{m}$ beneath the glass surface.

When focusing a laser beam into a sample of less than a-half-millimetre thickness, the challenge is to create refractive index modification close to the sample front surface rather than surface ablation. This limitation to writing useful structures is well noted and is highlighted in previous studies, such as the inscription of micro-channels through cylindrical fibre surface [24].

Hence, the laser processing conditions were studied in this paper, to achieve permanent index change structures in small dimensions $(\sim 3 \mu \mathrm{m}$ or less) within an undoped PMMA substrate. Particular emphasis will be placed on defining the laser writing parameters as a function of laser 
focus location below the surface. Writing structures under control at a range of focusing depth enables femtosecond laser micro-processing to be applied to situations where photonic structures are fabricated at variable depths below a curved surface such as a $125-\mu$ m-diameter single mode optical fibre.

\section{Laser modification theory}

During the experiments, laser refractive index modification was performed using transverse writing technology (Fig.1).

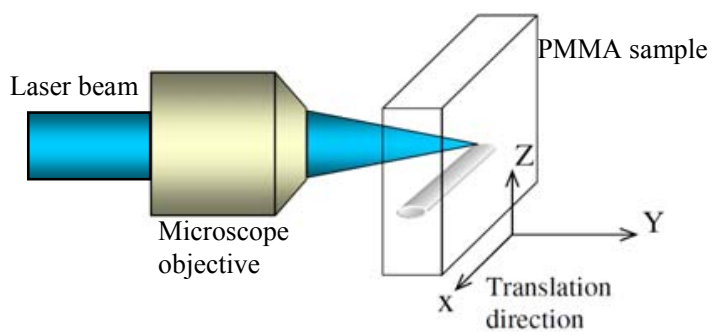

Fig.1 Schematic of transverse direct writing by fs laser. The laser beam propagates in the y-direction, with the sample translated in the $\mathrm{x}$-direction

When the material was irradiated by the laser pulses, the resultant refractive index change $(\Delta n)$ was due to the accumulated fluence, $\phi$ which was determined by the total number of incident laser pulses energy deposited to unit area $[25,26]$. If the laser beam linearly scans along the $x$ axis over a length $\mathrm{L}$, the accumulated laser fluence, $\phi$ can be calculated by equation (1), where $n_{S}$ denotes the number of overwrites, $n_{P}$ is the number of pulses per scanned line, $E_{p}$ is the laser energy per pulse, and $d_{s p}$ is the diameter of the focal spot in the material.

$$
\phi=\frac{n_{s} n_{p} E_{p}}{L \times d_{s p}}
$$

The number of pulses per scanned line $\left(n_{P}\right)$ is calculated by dividing the length of the line $(\mathrm{L})$, by the lateral distance of two pulses $\left(d_{P}\right) . d_{P}$ is given by the translation speed $\left(v_{S}\right)$ against the laser repetition rate (f). Therefore, equation (1) can be expressed in the form below

$$
\phi=\frac{n_{s} P_{a v}}{v_{s} \times d_{s p}}
$$

where $P_{a v}$ is the measured value of average laser power, giving $P_{a v}=E_{p} \times f$.

In order to maximise the refractive index change, $\Delta \mathrm{n}$, the incident laser fluence must be just below the material damage threshold. $\Delta \mathrm{n}$ was inferred by writing a diffraction grating and measuring the diffraction efficiency $\eta$ of a transmitted helium neon laser into the first order [15]. The magnitude of the refractive index modification was determined from $\eta$ using the equations for Raman-Nath diffraction of sub-surface phase grating in bulk material [27]. It had been demonstrated that the magnitude of the $\Delta \mathrm{n}$ modification was increased as a function of the laser fluence, but it was decreased if damage or filament formation took place [15].

\section{Experimental arrangement}

A Coherent Legend Ti: Sapphire laser facility (Photon Science Institute at the University of Manchester) generated horizontal linear polarised light, pulsed at a $1-\mathrm{kHz}$ repetition rate, with pulse duration of $100-\mathrm{fs}$ at a central wavelength of $800 \mathrm{~nm}$. The output beam from the system had a laser power of $\sim 3 \mathrm{~W}$ in the Gaussian mode with a diameter of $\sim 7 \mathrm{~mm}$. The schematic of the experimental setup is shown in Fig.2.

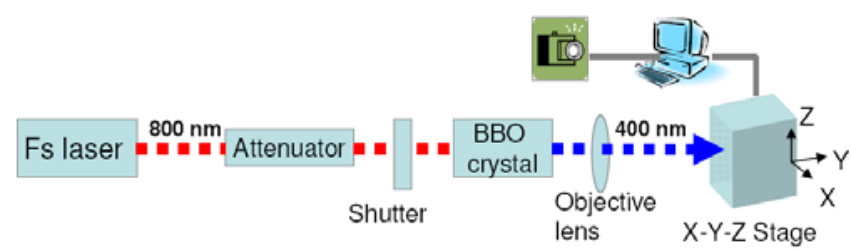

Fig.2 Schematic of experimental system

The laser beam was attenuated by a diffractive optic attenuator and its frequency was doubled by a BBO crystal, generating fs laser irradiation at $400 \mathrm{~nm}$ wavelength. This meant it had the potential to modify refractive index via two-photon absorption, resulting in larger $\Delta \mathrm{n}$ and smaller feature size of structural changes [17].

A neutral density (ND) filter placed between the reflection mirrors achieved a smaller integrated fluence. The laser beam was focused to $\sim 8 \mu \mathrm{m}$ diameter by a $20 \mathrm{x}$ Nikon microscope objective with a 0.45 numerical aperture (NA), $10 \mathrm{~mm}$ focal length and long working distance of $8 \mathrm{~mm}$. The single mode optical fibres and the bulk PMMA slabs were mounted on a PC controlled Aerotech $x-y-z$ translation stage (ANT-25LV) of $2.5 \mathrm{~nm}$ resolution and the fabrication process was viewed by a CCD Color Camera (DFK 31AF03).

The experiments were performed by translating the PMMA sample along the $\mathrm{x}$-direction so that the laser beam scribed parallel structures, line by line along $\mathrm{x}$ axis (as shown in Fig.1) inside the PMMA sheet of thickness 1-mm (Vistacryl CQ non UV). The femtosecond laser writing conditions were investigated by characterising the laser induced structures as a function of laser focal point location. The depth of the laser focal point below the sample surface varied from 10 to $500 \mu \mathrm{m}$. Since refractive index change, $\Delta \mathrm{n}$, rather than ablation or optical breakdown (Fig.3) was required, the influence of laser parameters and focusing conditions were studied to optimise pulse energy, lateral pulse distance and focusing depth within the material.

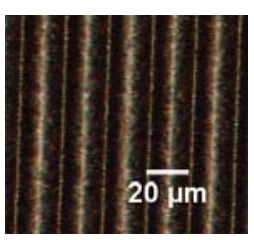

Material ablation

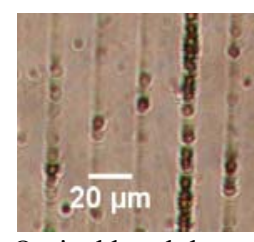

Optical breakdown

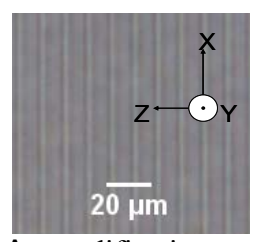

$\Delta \mathrm{n}$ modification
Fig.3 Microscope images of three stages of microstructure outcomes (top view)

The experiments using bulk PMMA samples involved two main steps of investigation: (1) the influence of the translation speed and the pulse energy at constant focusing 
depth (in y direction) of the laser beam into PMMA, (2) the structural dimension created at fixed pulse energy and translation speed whilst changing the focusing depth. The number of over scans was kept at unit for all modification areas throughout. The laser irradiated PMMA samples were cut in $\mathrm{y}-\mathrm{z}$ plane to make a cross section through the induced structures, and the cut surfaces were polished. The focusing depths $\left(\mathrm{F}_{\mathrm{d}}\right)$ discussed in the paper were measured from the beam focal point with respect to the surface along $y$ axis, i.e. the depth from the air-PMMA interface to the focal point of the laser beam below the PMMA surface.

A step-index single mode polymer optical fibre (POF) was also subjected to laser inscription. The POF (Paradigm Optics) has a $125 \mu \mathrm{m}$ PMMA cladding diameter and a $3.4 \mu \mathrm{m}$ PMMA core diameter. To remove the distortion effect of the curved fibre surface, the single mode POF was sandwiched between two flat PMMA sheets around which index-matching oil was injected as shown in Fig.4 and this setup was mounted mechanically on the $x-y-z$ stage[28].

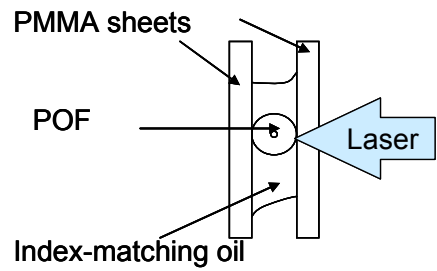

Fig.4 Schematic setup of fibre modification

\section{Results and analysis}

\subsection{Sub-surface microstructures written at various translation speeds and pulse energies}

Using transmitted light microscopy, the damaged or ablated region can be distinguished from the $\Delta \mathrm{n}$ region within the laser modified sample. The specific damage threshold as a function of pulse distance and pulse energy can be determined. The distance between two pulses was controlled by varying the laser writing velocity. Fig. 5 shows a plot of translation speed versus pulse energy in order to summarise the effect of these parameters for a $1 \mathrm{~mm}$ thick PMMA bulk material under various translation speeds ranging from $0.5 \mathrm{~mm} / \mathrm{s}$ to $3.0 \mathrm{~mm} / \mathrm{s}$, average laser energy up to $400 \mathrm{~nJ}$, and a fixed focusing depth of $100 \mu \mathrm{m}$ below the PMMA surface.

When the laser energy was reduced below $50 \mathrm{~nJ}$, no $\Delta \mathrm{n}$ modification was observed by inspection with an optical microscope. It implied that the laser irradiation was not sufficient to induce the photochemical change necessary to alter the material density, involving the direct cleavage of the polymer main chain, leading to a change in refractive index [16]. When applying low laser energy with slow translation speed, e.g., $60 \mathrm{~nJ}$ with $0.5 \mathrm{~mm} / \mathrm{s}$, the $\Delta \mathrm{n}$ modification took place. It was observed that the damage threshold for PMMA reduced with increased incident laser energy level. These thresholds were $1.41,0.82,0.63,0.53$, and $0.47 \mathrm{Jcm}^{-2}$ for corresponding pulse energy of $\mathrm{E}_{\mathrm{p}}=60,70$, 80,90 , and $100 \mathrm{~nJ}$ respectively. This indicates that $\Delta \mathrm{n}$ is maximised by using multiple overwrites at low fluences. This phenomenon was previously observed when multiple overwrites at low laser fluence at short pulses $(<100 \mathrm{fs})$, achieved more efficient modification, which was larger $\Delta \mathrm{n}$, via cumulative incubation reactions $[17,29]$. To summarise, for $100 \mathrm{fs}$ laser pulses focused at a constant depth inside the sample using a lower pulse energy with the same number of pulses irradiating a defined area could enlarge the $\Delta \mathrm{n}$ modification range without reaching the damage threshold.

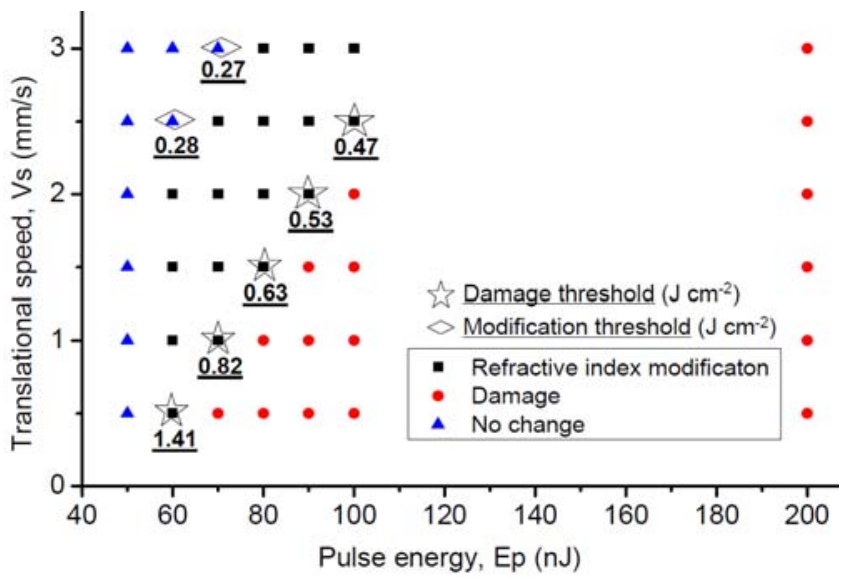

Fig.5 Damage and modification produced by varying $\mathrm{v}_{\mathrm{S}}$ and $E_{p}$ at a constant $F_{d}=100 \mu \mathrm{m}$.

Laser energies above 200nJ caused inner material damage or surface ablation for $\mathrm{v}_{\mathrm{S}}$ in the range $0.5-3.5 \mathrm{~mm} / \mathrm{s}$, so this should be avoided in refractive index structural writing. Single-pulse irradiation was observed if the sample was translated faster than $3 \mathrm{~mm} / \mathrm{s}$ due to the $1-\mathrm{kHz}$ repetition rate generating a broken line, and no continuous structural change could be produced above this speed.

The cross-sectional shape of each scanned line was expected to be elongated at the focal region along the $\mathrm{y}$ axis due to the self-focusing effect. Optical images of the cross sections presented in the $y-z$ plane in Fig. 6 were observed by an optical microscope from the $\mathrm{x}$ axis direction. The laser beam was focused into the PMMA sample with the focus point located $\sim 15 \mu \mathrm{m}$ beneath the surface. The crosssection along the $\mathrm{x}$ direction ranges from approximately $40 \mu \mathrm{m}$ (Fig.6a) down to $2 \mu \mathrm{m}$ (Fig.6d). Although the crosssectional shapes exhibited an elliptical feature, the spatial shape became relatively small with reduced laser energy. In addition, elongation of the resultant structural cross section was not affected by the stage translation speeds ranging from 0.5 to $2.5 \mathrm{~mm} / \mathrm{s}$ despite the same energy being delivered.

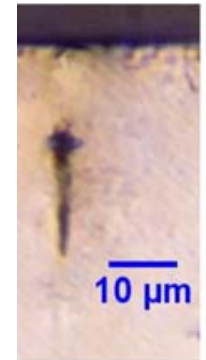

(a)

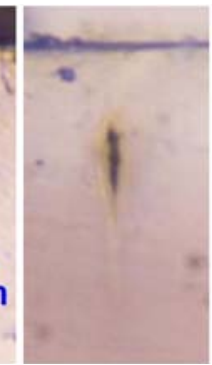

(b)

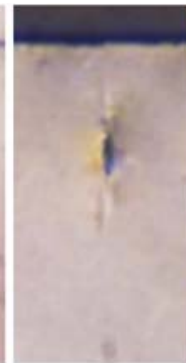

(c)

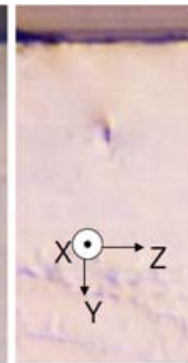

(d)
Fig.6 Optical microscope images of the cross-sectional shape of inscribed structures. The incident energies $\mathrm{E}_{\mathrm{p}}$ were (a) $250 \mathrm{~nJ}$, (b) $200 \mathrm{~nJ}$, (c) $100 \mathrm{~nJ}$ and (d) 70 nJ, with $\mathrm{v}_{\mathrm{S}}=1 \mathrm{~mm} / \mathrm{s}$.

A low fluence level of $0.82 \mathrm{~J} \mathrm{~cm}^{-2}(70 \mathrm{~nJ}$ and $1 \mathrm{~mm} / \mathrm{s})$ initiated the $\Delta \mathrm{n}$ modification at $\mathrm{F}_{\mathrm{d}}=100 \mu \mathrm{m}$ as shown in 
Fig.5. However, the irradiated regions shown in Fig.6 exhibit optical breakdown due to a low focusing depth of $\sim 15 \mu \mathrm{m}$ even if the incident fluence used was the same. This indicated that the $\Delta \mathrm{n}$ modification is also affected by the laser beam focal point location.

\subsection{Effect of focusing depth in bulk PMMA}

The effect of laser beam focus depth was studied by

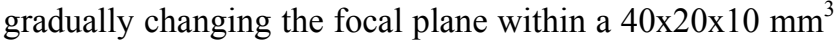
PMMA sheet, while keeping the translational speed at a constant of $v_{S}=1 \mathrm{~mm} / \mathrm{s}$. The width (w) and the depth (d) of cross sections of induced structures were measured referred to the $\mathrm{z}$ axis and $\mathrm{y}$ axis, and were plotted as a function of focusing depth $\left(\mathrm{F}_{\mathrm{d}}\right)$ of the laser beam inside PMMA in Fig. $7 \mathrm{a}$ and Fig. $7 \mathrm{~b}$ respectively.

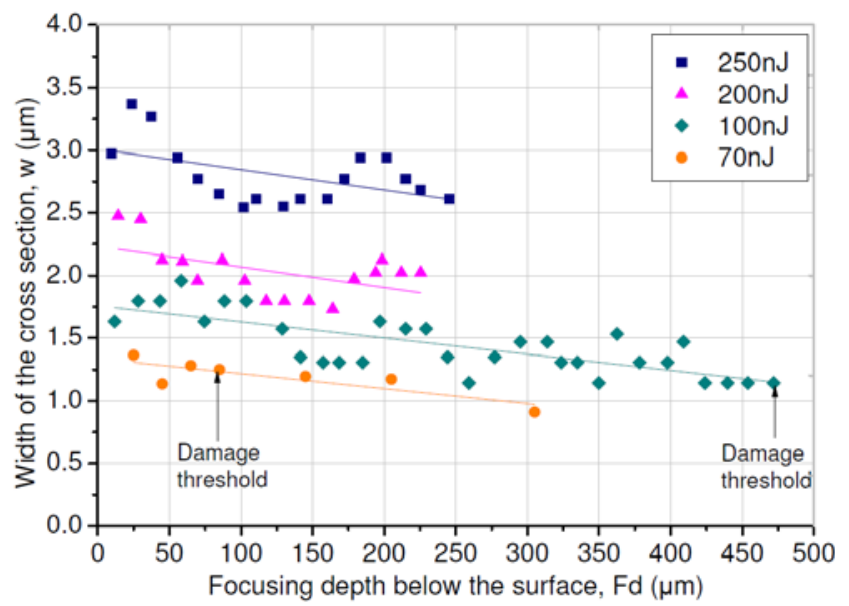

(a)

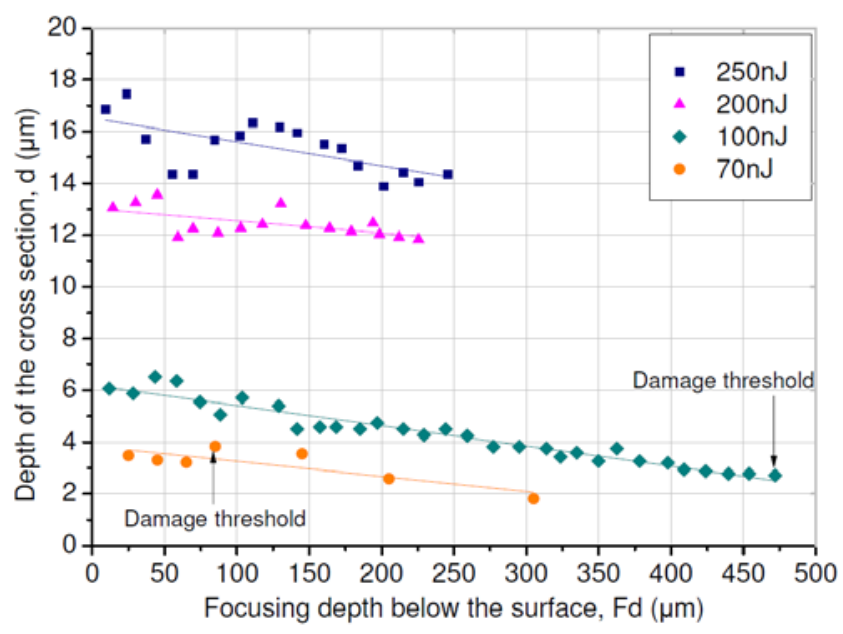

(b)

Fig.7 (a) Width and (b) Depth of the cross-sectional structures as a function of the focusing depth below the PMMA surface. $\left(\mathrm{v}_{\mathrm{S}}=1 \mathrm{~mm} / \mathrm{s}\right)$

The average laser pulse energy was in the range from $70 \mathrm{~nJ}$ to $250 \mathrm{~nJ}$. At the focusing depth of $\mathrm{F}_{\mathrm{d}}=\sim 90 \mu \mathrm{m}$, refractive index structures were produced with a pulse energy of $E_{p}=70 n J$. However, this energy was insufficient to create a change in refractive index at $F_{d}=470 \mu \mathrm{m}$, unless $E_{p}$ reached $100 n J$. When $E_{p}>200 n J$, the structural change observed below the damage threshold was in the deep regions of $F_{d}$ $>\sim 700 \mu \mathrm{m}$. Thus, increasing focusing depth within the submillimetre PMMA sheet resulted in increased damage threshold, similar to the observation by Liu [23] for inscribing waveguides in silica glass.

The cross sections dimension decreased with increased beam focused depths, implying the absorbance at the UV writing wavelength $(400 \mathrm{~nm})$ increased with increasing thickness of the PMMA sample as would be expected from the Beer-Lambert Law [30]. Along each trend line, the measured data exhibited periodic oscillations in the values of the cross-section dimensions. The amplitude of the oscillation increased with increasing laser fluence, but it decreased when the laser focal plane was moved deeper into the PMMA sample. These variations may be caused by instabilities of intensities in fs laser intensities at the focal point, attributed to non-linear absorption and non-linear propagation effects as the input energy is increased [31-33].

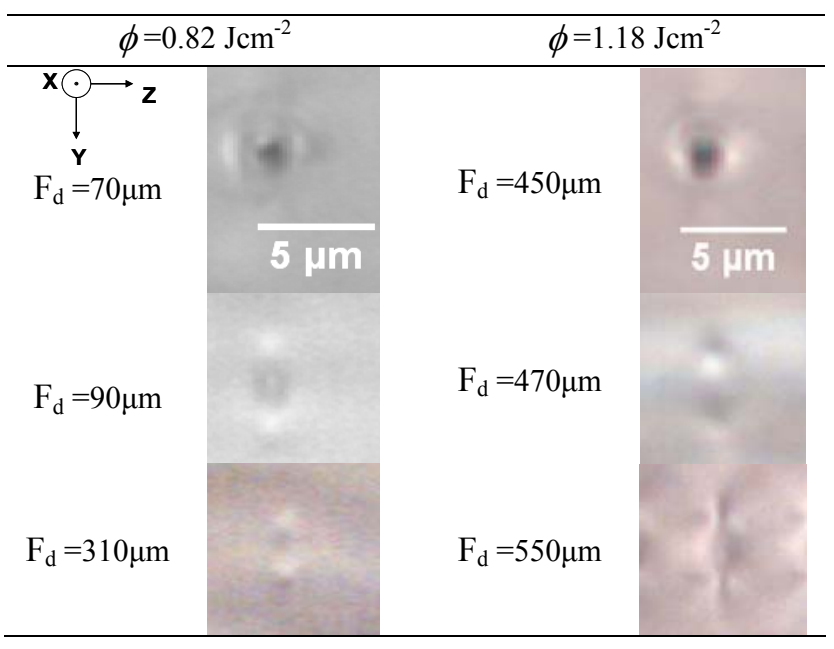

Fig.8 Cross section view of outcomes of laser irradiation with fluence of $0.82 \mathrm{Jcm}^{-2}\left(E_{\mathrm{p}}=70 \mathrm{~nJ}, \mathrm{v}_{\mathrm{S}}=1 \mathrm{~mm} / \mathrm{s}\right)$ and $1.18 \mathrm{Jcm}^{-2}\left(\mathrm{E}_{\mathrm{p}}=100 \mathrm{~nJ}, \mathrm{v}_{\mathrm{S}}=1 \mathrm{~mm} / \mathrm{s}\right)$ at different focusing depths $\left(\mathrm{F}_{\mathrm{d}}\right)$.

Typical microscope images of scanned line cross sections with incident fluence of 0.82 and $1.18 \mathrm{Jcm}^{-2}$ were showed in Fig.8. The damage threshold for using $0.82 \mathrm{Jcm}^{-2}$ was observed at a depth of $F_{d}=70 \mu \mathrm{m}$. When $F_{d}$ was changing to $90 \mu \mathrm{m}, \Delta \mathrm{n}$ modification occurred. No refractive index change was observed if $F_{d}>350 \mu \mathrm{m}$. The refractive index structure was observed in the region of $90 \mu \mathrm{m}<\mathrm{F}_{\mathrm{d}}<350 \mu \mathrm{m}$, and the dimension of the cross sections $(\mathrm{w}=\sim 1 \mu \mathrm{m}, \mathrm{d}=2$ $4 \mu \mathrm{m})$. When an increased laser fluence of $1.18 \mathrm{Jcm}^{-2}$ was used, $\Delta \mathrm{n}$ modification commenced at a deeper region $\left(\mathrm{F}_{\mathrm{d}}=\right.$ $470 \mu \mathrm{m})$. Increasing the writing depth to $550 \mu \mathrm{m}$, the cross section shape was elongated more along the beam propagation direction, because the non-linear effect dominated and filamentation was stronger with increased focusing depths [34]. On the other hand, if the focal point increased to more than $550 \mu \mathrm{m}$, the feature size of the fabricated structures cross section is not reduced and not suitable for waveguide fabrication. It was concluded that lower laser fluence, avoiding the enhanced filamentation effect, should produce refractive index structures with relatively small feature of cross-sectional shape over a larger range of the writing depth. 
The cross-section profile was demonstrated to be circular in shape by using the longitudinal writing technique in early studies $[35,36]$. However, this method is limited by the working distance of the focusing objective used. The structural dimension induced by transverse writing technology, was reduced to focusing depths up to $500 \mu \mathrm{m}$ with low laser energies in this study. Consequently, to overcome the difficulty of fabricating inner 3D refractive index structures due to the limitation of small thickness $(<500 \mu \mathrm{m})$ or irregular sample geometry, sandwiching the sample between two planar specimens from the same material offers a feasible method for structuring.

\section{3 $\Delta$ n modification in POF}

Understanding the feature size dependence on the focusing depth and the laser fluence, enables the possibility of writing 2-D and 3-D optical structures located at varied depths beneath the surface, by appropriate adjustment of processing conditions. To fabricate the long period grating (LPG) in PMMA based POF, the POF was sandwiched between two flat PMMA sheets around which indexmatching oil was injected. Since the fibre acts as a cylindrical focusing optic, the laser modification product combined a structural distortion in focal volume due to the fibre cylindrical surface, leading to the mismatch of the focal plane. The mismatched optical path of the laser beam focused position in POF resulted from the change in refractive index from air $(n=1)$ to POF $\left(n^{\prime}=\sim 1.5\right)$ interface. Applying the matching oil of $n=1.5$ between two PMMA sheets enabled precise laser modification with a flat interface and continuous inner material index. For pulse energy of $100 \mathrm{~nJ}$, the refractive index modification occurred at a focusing depth of $470 \mu \mathrm{m}$ below the PMMA surface, producing a structural change with a $3 \mu \mathrm{m}$ by $1.3 \mu \mathrm{m}$ for cross section. As a result, two $500 \mu \mathrm{m}$ thick PMMA sheets were selected for experiments. An incident fluence level of $\phi=1.1 \mathrm{Jcm}^{-2}$ was used, since this fluence was just below the damge threshold for writing $\Delta \mathrm{n}$ structures into the POF.
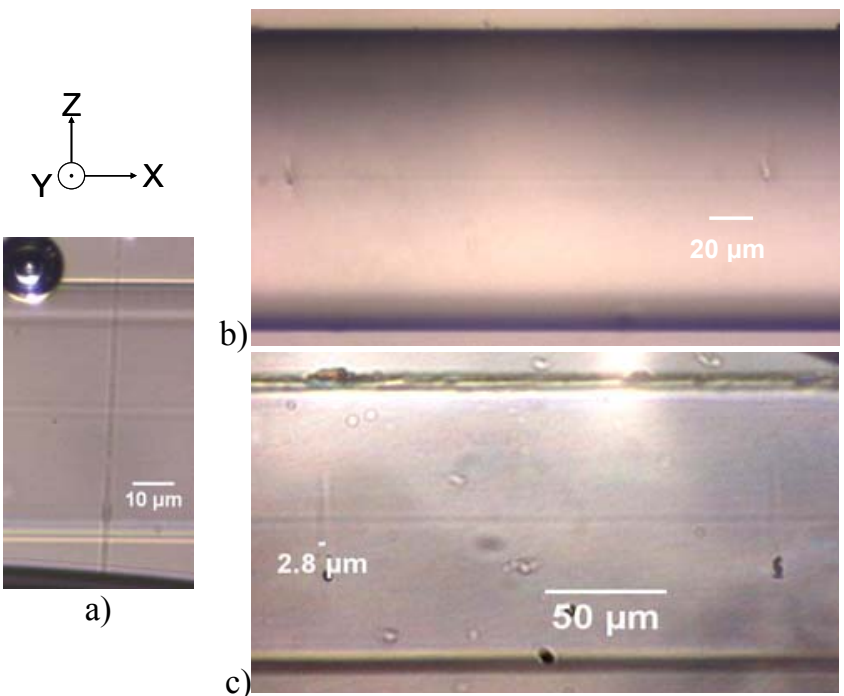

Fig.9 A section of LPG demonstrates (a) the continuous inner structural change induced by fs lasers within the sandwich setup. A part of in-fibre grating is shown in (b) cross view and (c) side view.
It is demonstrated in Fig.9a that this setup enabled precise laser micro-structuring with flat interface and continuous inner material. A refractive index grating having a period of $200 \mu \mathrm{m}$ was written successfully throughout the fibre core and observed by the microscope (Fig.9b-9c). The individual $\Delta \mathrm{n}$ structure width measured was $2.8 \mu \mathrm{m}$. The size of the cross-sectional structure may be reduced by using a thinner PMMA sandwich configuration with reduced laser fluence.

Long period gratings (LPG) with grating periods of 100 , $150,175,200$ and $250 \mu \mathrm{m}$, were inscribed respectively into five Paradigm POFs, and all LPGs had grating lengths of $20 \mathrm{~mm}$. The transmission spectra for LPGs surrounded by air were measured using an Ocean Optics USB4000 fibre optic spectrometer $(350-1000 \mathrm{~nm}$, optical resolution $\sim 1.5 \mathrm{~nm}$ at FWHM). A LS-1 Tungsten Halogen Light Source (Ocean Optics) optimised for the visible-near Infrared was used as a light source, coupled to the single mode POF via SMA connectors.

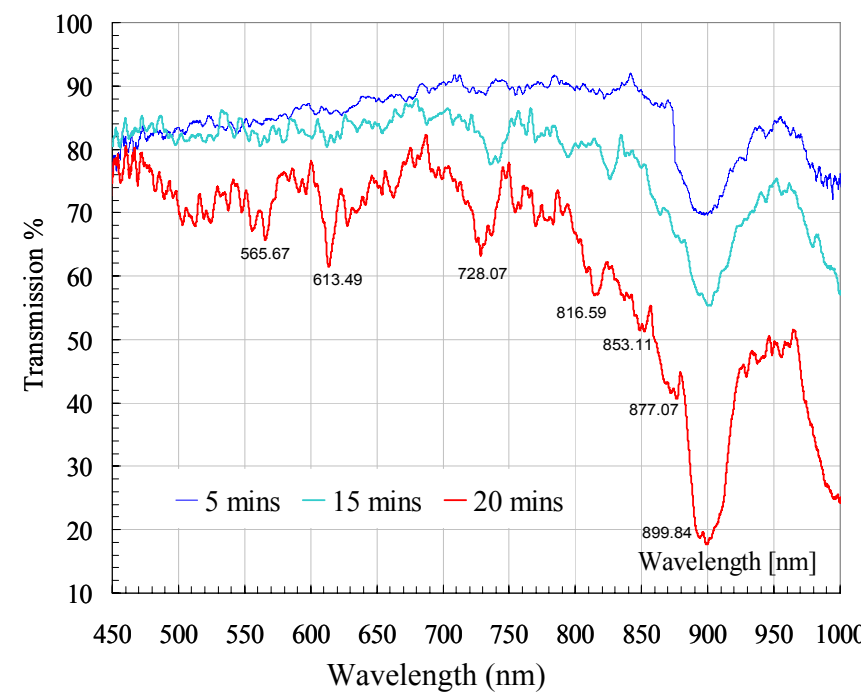

Fig.10 Change in transmission of a $250 \mu \mathrm{m}$ period LPG as a function of time.

In the visible spectrum, the attenuation bands observed will only be generated by a LPG with a period of $250 \mu \mathrm{m}$, since PMMA itself shows no spectral features in this region[12]. The change in transmission loss of LPG written with $250 \mu \mathrm{m}$ period as a function of time is shown in Fig. 10. At the first five minutes after the completion of laser irradiation, small changes in transmission $(<5 \%)$ were observed ranging from 450 to $850 \mathrm{~nm}$. After 15 minutes, the loss in transmission increased at the wavelength of 730 and $820 \mathrm{~nm}$ particularly, having a difference of $\sim 10 \%$ and $\sim 6 \%$. Twenty minutes later, the attenuation peaks reached their maximum value and there was no further change in transmission. The resonance wavelengths were optimised with a time delay after irradiation by the fs laser, possibly due to a photochemical process and a thermal reaction [16]. The LPG produced an initial attenuation band that had resonance wavelengths of around $565 \mathrm{~nm}$, and other attenuation bands were generated at $613,728,816,853,877$ and 900nm. 


\section{Conclusion}

The dependence of laser writing depth, pulse spacing as well as optimised writing fluence in PMMA demonstrated the feasibility of fabricating 3-D internal optical components within the PMMA substrate less than a millimetre thick. Highly localised refractive index structures were generated at a controlled spatial resolution written into bulk PMMA. The feature size of the cross-sectional shape was $2 \mu \mathrm{m}$ by $0.8 \mu \mathrm{m}$ and $3 \mu \mathrm{m}$ by $1.3 \mu \mathrm{m}$ observed at a depth of $300 \mu \mathrm{m}$ and $500 \mu \mathrm{m}$ below the surface. This practical demonstration indicated precise location of features across the small core $(3.4 \mu \mathrm{m}$ diameter) of a single mode polymer fibre. The effect of the curved fibre surface has been removed, and the laser inscription spatially controlled using index matching oil and sandwich arrangement. Further work will be carried out to evaluate the dependence of absorbance near UV wavelength at different focusing depth and to investigate the limitation of multi-channel curved waveguides and internal optical connecters in PMMA. Further applications will include integrated optic circuits, optically interrogated micro-fluidics systems and optical interconnects.

\section{Acknowledgments}

The authors gratefully acknowledge help from Professor Lin Li and Dr. David Whitehead of MACE at the University of Manchester. Shijie Liang is supported by ORS and CEAS Studentship.

\section{References}

[1] C.R. Mendonca, L.R. Cerami, T. Shih, R.W. Tilghman, T. Baldacchini and E. Mazur: Optics Express, 16, (2008) p.200-206.

[2] N. Uppal, P.S. Shiakolas and M. Rizwan: Proc. of Micromachining Microfabrication Process Technology XIII, San Jose CA, (2008) p.I8820-I8820.

[3] W. Watanabe: Laser Physics, 19, (2009) p.342-345.

[4] C. Wochnowski, Y. Cheng, K. Meteva, K. Sugioka, K. Midorikawa and S. Metev: J. Optics a-Pure and Applied Optics, 7, (2005) p.493-501.

[5] K. Kalli, H.L. Dobb, D.J. Webb, K. Carroll, M. Komodromos, C. Themistos, G.D. Peng, Q. Fang, I.W. Boyd: Opt. Lett., 32, (2007) p.214-216.

[6] C. Florea and K.A. Winick: Journal of Lightwave Technology, 21, (2003) p.246-253.

[7] G. Della Valle: Journal of Optics a-Pure and Applied Optics, 11, (2009) 1.

[8] K. Sugioka, Y. Hanada and K. Midorikawa: Applied Surface Science, 253, (2007) 6595-6598.

[9] D.F. Farson, H.W. Choi, C.M. Lu, and L.J. Lee: J. Laser Applications, 18, (2006) 210-215.

[10] R.M. Vazquez, R. Osellame, M. Cretich, M. Chiari, C. Dongre, H. Hoekstra, M. Pollnau, H. van den Vlekkert, R. Ramponi, G. Cerullo: Analytical and Bioanalytical Chemistry, 393, (2009) 1209-1216.

[11] R.Q. Frazer, R.T. Byron, P.B. Osborne and K.P. West: J. Long Term Eff Med Implants, 15, (2005) 629-639.

[12] O. Ziemann, J. Krauser, P. Zamzow and W.D. Berlin: "POF Handbook: Optical Short Range Transmission Systems", 2nd ed., Springer, 2008.
[13] J.E. Prest, P.R. Fielden, N.J. Goddard and B.J.T. Brown: Measurement Science \& Technology, 19, (2008).

[14] J.E. Prest, S.J. Baldock, P.R. Fielden, N.J. Goddard and B.J.T. Brown: Analyst, 127, (2002) p.1413-1419.

[15] P.J. Scully, D. Jones, D.A. Jaroszynski: J. Optics A: Pure and Applied Optics 5 (2003) S92-S96.

[16] A. Baum, P.J. Scully, M. Basanta, C.L.P. Thomas, P.R. Fielden, N.J. Goddard, W. Perrie and P.R. Chalker: Opt. Lett., 32, (2007) p.190-192.

[17] A. Baum, P.J. Scully, W. Perrie, D. Jones, R. Issac and D.A. Jaroszynski: Opt. Lett., 33, (2008) p.651-653

[18] A. Baum, P.J. Scully, W. Perrie, M. Sharp, K.G. Watkins, D. Jones, R. Issac, D.A. Jaroszynski: Proc. 7th Int. Symp. on Laser Precision Microfabrication, Vienna, Austria (2007).

[19] W. Liu, and S.L. Chin: Science in China Series E: Technological Sciences 50 (2007) 413-421.

[20] W. Watanabe, T. Tamaki and K. Itoh: Laser Physics, 18, (2008) p.263-269.

[21] A. Brodeur and S.L. Chin: J. OSA B-Optical Physics, 16, (1999) p.637-650.

[22] A. Saliminia, N.T. Nguyen, S.L. Chin and R. Vallee: Optics Comm., 241, (2004) p.529-538.

[23] D. Liu, Y. Li, R. An, Y. Dou, H. Yang and Q. Gong: Applied Physics a-Materials Science \& Processing, 84, (2006) 257-260.

[24] D. Day and M. Gu: Optics Express, 13, (2005) 59395946.

[25] J. Schille, R. Ebert, U. Loeschner, L. Schneider, N. Walther, P. Scully, N. Goddard and H. Exner: Proc. 5th Int. Symp. on Lasers in Manufacturing, Munich, (2009).

[26] W. Perrie, M. Gill, G. Robinson, P. Fox, W. O'Neill, Applied Surface Science 230 (2004) 50-59

[27] H. Kogelnik: Bell System Technical Journal, 48, (1969) p.2909-2947.

[28] S.J. Liang, P.J. Scully, J. Schille, J. Vaughan and W. Perrie: Proc. 20th Int. Symp. on Optical Fibre Sensors, SPIE, (2009) p.75036S.

[29] A. Baum, P.J. Scully, W. Perrie, D. Liu, V. Lucarini: J. Opt. Soc. Am. B 27 (2010) 107-111.

[30] J.D.J. Ingle, S.R. Crouch: "Spectrochemical Analysis", (Prentice Hall, New Jersey, 1988)

[31] M.S. Sodha, R.P. Sharma and V.K. Tripathi: J. Appl. Phys., 7, (1974) p.2471-2478.

[32] P., W. Wasilewski, M. Matuszewski, M. Trippenbach, C. Radzewicz: Proc. Spie-Int Soc Optical Engineering Bellingham, (2002), 20-24.

[33] M. Centurion: "Study of the nonlinear propagation of femtosecond laser pulses", California Institute of Technology, California, (2005).

[34] Q. Sun, H.B. Jiang, Y. Liu, Y.H. Zhou, H. Yang and Q.H. Gong: J. Optics a-Pure and Applied Optics, 7, (2005) 655-659.

[35] A. Saliminia, N.T. Nguyen, M.C. Nadeau, S. Petit, S.L. Chin and R. Vallee: J. Appl. Phys., 93, (2003) p.3724-3728.

[36] C. Mauclair: A. Mermillod-Blondin, N. Huot, E. Audouard and R. Stoian: Optics Express, 16, (2008) p.5481-5492. 